\title{
Empleabilidad y empresas de inserción: modelo de prestaciones y apoyos
}

\author{
Natxo Martínez-Rueda
}

Departamento de Pedagogía Social y Diversidad, Universidad de Deusto

natxo.martinez@deusto.es

\section{Javier Galarreta Lasa}

Departamento de Didáctica y Organización Escolar, Universidad del País Vasco/Euskal Herriko Unibertsitatea (UPV/EHU)

javier.galarreta@ehu.es

\section{Igone Aróstegui Barandica}

Departamento de Didáctica y Organización Escolar, Universidad del País Vasco/Euskal Herriko Unibertsitatea (UPV/EHU)

igone.arostegui@ehu.eus

Artikulu honetan, Gizatea-Gizarteratzeko eta Laneratzeko Euskadiko Enpresen Elkarteko profesionalekin izandako elkarrizketa-prozesuaren eta kontrastearen ondorioz laneratze-laguntza proposamena aurkezten da Laneratzeko Enpresen orientaziorako. Enpleguko politika aktiboen baitan dagoen enplegagarritasunaren kontzeptuaren azterketaren ondorioz kontzeptuaren ikuspegi zabala eta elkarreragilea proposatzen da. Halaber, Laneratzeko Enpresen enplegagarritasunaren kontzeptuaren eredua eraikitzen da, non banakako eta testuinguruko osagaiak bi tresnen bitartez neur daitezke. Era berean, Laneratzeko Enpresek garatzeko laguntza-arloak eta prestazio batzuk proposatzen dira lan-merkatuan sartzeko zailtasun bereziak dituzten pertsonen enplegagarritasuna hobetzeko.

\section{GAKO-HITZAK:}

Laneratzeko Enpresak, enplegagarritasuna, laneratzea, prestazioak.
En este artículo se presenta, producto de un proceso de diálogo y contraste con profesionales de Gizatea-Asociación de Empresas de Inserción del País Vasco, una propuesta para orientar el trabajo de acompañamiento en las empresas de inserción. A partir de un análisis crítico del concepto de empleabilidad implícito en las políticas activas de empleo, se propone una visión amplia e interactiva de este y se construye un modelo de empleabilidad en las que incorpora elementos tanto individuales como organizacionales que se operativizan a través de dos herramientas. En base a lo anterior, se aporta una propuesta de las áreas de apoyo y prestaciones que pueden desarrollar las empresas de inserción para mejorar la empleabilidad de personas con especiales dificultades de acceso al empleo.

\section{Palabras CLAVE:}

empresas de inserción, empleabilidad, acompañamiento, prestaciones. 


\section{Introducción}

Las empresas de inserción (EI) son una realidad consolidada en nuestro contexto, con 42 empresas en el País Vasco asociadas a Gizatea-Asociación de Empresas de Inserción del País Vasco, que dan empleo a 1.162 personas, de las cuales 702 estaban en procesos de inserción (Gizatea, 2018). Sus resultados, con altas tasas de incorporación al empleo -el 69\% en 2017-, están evidenciando que son un programa eficaz para responder a las necesidades de personas con necesidades complejas de inserción laboral.

Desde su creación, y respondiendo a su misión, Gizatea ha promovido en el sector dinámicas de reflexión, análisis, formación y sistematización de procesos de trabajo en colaboración con la universidad (Martínez y Alvarez de Eulate, 2015). Durante cerca de quince años, esta colaboración ha permitido desarrollar un conjunto de materiales (Fernández, Galarreta, y Martínez, 2007; Gallastegi y Martínez, 2011; Martínez, Gallastegi y Yániz, 2012) que han servido de referencia a sus profesionales para desarrollar y mejorar sus prácticas profesionales.

Uno de estos materiales, el Manual de Acompañamiento en las Empresas de Inserción (Fernández et al., 2007) ha sido revisado durante 2018 por un equipo de trabajo compuesto por profesionales ${ }^{1}$ y académicos para responder a los cambios producidos en el contexto y para recoger la experiencia acumulada durante este tiempo. En este proceso, una parte del trabajo se ha centrado en el análisis y revisión del modelo de acompañamiento de Gizatea. Producto de este trabajo se han desarrollado, explicitado y sistematizado algunos de sus componentes con el objetivo de enriquecer el marco de referencia desde el que están operando las El en su vertiente de acompañamiento.

El objetivo de este artículo es presentar, como resultado de este proceso, un modelo de referencia para el desarrollo de los procesos de acompañamiento en las empresas de inserción. Para ello se discute brevemente la crisis del empleo y las implicaciones que tiene para las El y se revisan algunas limitaciones de las políticas de empleo que nos llevan a rescatar el concepto de empleabilidad como un concepto dinámico y relativo. Sobre estas referencias se definen algunos de los rasgos de las El y se propone un modelo de empleabilidad en ellas que incorpora factores tanto contextuales como personales, así como las herramientas que facilitan su evaluación. En la última parte del artículo, y tomando como referencia tanto la normativa como los diferentes ámbitos y

${ }^{1}$ Leire Álvarez de Eulate (Gizatea), Susana Porras (Cáritas Bizkaia), Pepe San José (Koopera Servicios Ambientales), Haizea Roca (Sokaire, Keima Animazioa), Susana Albaina (Berezi 99), Jon Sierra (Emaús Dendak), María Larrinaga (Servicios de Hostelería Peñascal, Iturritek), Miriam Santorcuato (Suspertu), Jesús Gazapo (Ikatzbizi), César Sardón (Urbegi Inserción) y Maitane Burgos (Zabaltegi). oportunidades que conforman la oferta de las El, se hace una propuesta de las prestaciones y apoyos que tienen que desarrollarse desde las empresas.

\section{Contexto y referencias para el acompañamiento en las empresas de inserción}

Las empresas de inserción como un recurso dirigido a la inserción laboral de personas en situación o riesgo de exclusión tienen que considerar el contexto socioeconómico y político en el que se desenvuelven. En este sentido, hay algunos elementos que caracterizan la situación actual y que se articulan en torno a la crisis del empleo como mecanismo de incorporación social que es preciso identificar para ajustar las respuestas.

Las profundas transformaciones que se están produciendo del paso de la sociedad industrial a la sociedad tecnológica, informacional o del conocimiento, implican un cambio sustancial en los patrones de organización social. Este proceso de cambio está generando también diferentes formas de desigualdad, pobreza y vulnerabilidad social, en el marco de "sociedades duales". En la sociedad industrial, el empleo era el principal factor de inserción y, por tanto, la puerta de entrada a la organización social, en la medida en que no solo permitía unos ingresos económicos o el acceso a la protección social que aseguran la existencia, sino que también garantizaba experiencias sociales básicas (Beck, 1998) como identidad, pertenencia, contribución o relaciones sociales.

Aunque el empleo sigue siendo el mecanismo fundamental para facilitar la incorporación social, hoy por sí mismo, no la garantiza en la medida en que estamos en "un sistema lleno de riesgos de subempleo más flexible, plural y descentralizado" (Beck, 1998: 180). Incluso, las importantes segmentaciones del mercado de trabajo permiten hablar de "empleos de exclusión" (Foessa, 2014). Por otra parte, también hay que tener en cuenta que el incremento del desempleo de larga duración y la progresiva precarización de una parte del empleo son tendencias presentes en la evolución actual de los mercados laborales occidentales (Pérez Eransus, 2015).

En resumen, la cadena básica en el capitalismo industrial se ve cuestionada en el capitalismo informacional, desvaneciéndose la idea de una conexión más o menos directa entre acceso a un empleo e inclusión social. En el futuro, el empleo va a escasear y, en muchos casos, no va a asegurar la inserción social, sobre todo en niveles bajos de cualificación. A pesar de estos profundos cambios y de que el empleo ha reducido su capacidad de integración, y tendrá que ser compensado con otros mecanismos de incorporación social -rentas básicas, revalorización de otras actividades, etc.-, 
preparar y apoyar el acceso al empleo seguirá siendo un aspecto central en las políticas de inclusión.

Con sus contradicciones, el empleo sigue siendo un elemento necesario para muchas personas y es altamente valorado socialmente.

En este sentido, y con las limitaciones señaladas, el empleo remunerado sigue siendo en nuestros días un mecanismo esencial de inclusión social, de realización personal y de protección contra la pobreza. Carecer de empleo incrementa entre tres y cinco veces el riesgo de estar en una situación de exclusión (SIIS, 2011). En la medida en que el empleo no garantiza ni inclusión ni calidad de vida, aunque sea una herramienta para ello, es necesario desarrollar una visión amplia de inclusión con sus apoyos correspondientes y monitorizar la relación entre el empleo y la calidad de vida de las personas. En este sentido, resulta relevante atender al impacto del empleo en las diferentes dimensiones de calidad de vida (Schalock y Verdugo, 2003; Blanco, Kortajarena y Romero, 2015) de las personas: bienestar material, bienestar físico, bienestar emocional, inclusión social, relaciones sociales, desarrollo personal, derechos y autodeterminación.

Este contexto tiene algunas implicaciones que las El tienen que considerar en sus procesos de acompañamiento. En primer lugar, la necesidad de buscar conexiones entre el hecho de disponer de un empleo y la calidad de vida de las personas, evaluando el impacto que tiene en la calidad de vida de una persona su participación en una El. En este sentido, incorporar la calidad de vida como medida multidimensional de la inserción (Donoso y Figuera, 2007) puede tener diversas implicaciones en la acción y la evaluación de las El. Por ello, va a ser necesario proyectar itinerarios con una mirada global que incorporen el empleo, pero que miren también más allá de él. Otra derivada del contexto actual del empleo se relaciona con la importancia de enfatizar tanto en las evaluaciones de resultados como en el desarrollo de los itinerarios la idea de "empleo de calidad". No vale cualquier inserción y las El se deben orientar a empleos de calidad suficiente.

Por último, muy importante también es la exigencia de enfatizar la promoción personal, social, cultural y laboral de las personas a través de la formación general, la cualificación profesional, el desarrollo de la autodeterminación y la vinculación y participación social. Estos ingredientes en el contexto de crisis del empleo son imprescindibles.

\subsection{Políticas activas de empleo y empleabilidad}

La magnitud de las transformaciones que se están produciendo, con la consiguiente complejidad de los problemas que se generan, ya nos hablan de la necesidad de compaginar análisis y planteamientos críticos globales - para no ser ingenuos en nuestras actuaciones- con propuestas concretas y posibilistas para poder influir en las realidades concretas. En el marco del escenario dibujado, es fácil de deducir las limitaciones de las políticas activas de empleo para responder al problema del desempleo y sus consecuencias. A pesar de que el debate general debe ampliarse a lo que se ha denominado "medidas alternativas" (Santos Ortega, 2002) en el marco de lucha contra la exclusión (servicios de calidad, reparto del trabajo, rentas básicas, etc.) las políticas activas de empleo siguen siendo, en nuestro contexto, la referencia para desarrollar propuestas y medidas a corto plazo. Estas medidas, sabiendo que son limitadas y que no resuelven el problema de fondo de carácter estructural, permiten, por una parte, mejorar la situación de determinadas personas y colectivos y, por otra, ensayar nuevos modelos de organización social, de carácter inclusivo.

Al mismo tiempo, resulta importante reconocer que las políticas activas de empleo, en sintonía con el paradigma de la activación, se han orientado frecuentemente hacia la modificación de las conductas, motivaciones y actitudes de las personas, más que a la intervención en las condiciones contextuales que generan desempleo y exclusión (SIIS, 2011). Es habitual, de forma implícita, responsabilizar a las personas de su situación: falta de habilidades, de cualificación, situaciones personales, etc. Mejorar la empleabilidad desde esta perspectiva es resultado del esfuerzo personal, fundamentalmente de formación, pero también de motivación y adaptación.

En síntesis, tenemos que reconocer que, a pesar de que la empleabilidad depende tanto del contexto como de los sujetos, las políticas de empleo se han centrado más en los sujetos - con mayores exigencias-con el consiguiente riesgo de culpabilización de las personas. Frente a este planteamiento, parece necesario rescatar una comprensión amplia del concepto de empleabilidad que ayude a mantener un equilibrio entre responsabilidad individual y responsabilidad social, entre mejora de las competencias y actitudes y el desarrollo de entornos accesibles con variedad de oportunidades.

\subsection{Revisando el concepto de empleabilidad}

El término "empleabilidad" tiene una larga tradición, con múltiples sentidos y usos (Serrano Pascual, 2000). Para algunos se ha convertido en un concepto confuso (De Grip, Loo y Sanders, 2004) al haber ido incorporando de forma progresiva nuevos elementos y componentes, coexistiendo diferentes definiciones (Williams et al., 2015). El concepto actual implica una visión amplia y dinámica de la empleabilidad y debe abarcar factores tanto individuales como contextuales (De Grip et al., 2004; McQuaid y Lindsay, 2005), en una conceptualización cada vez más compleja y multidimensional (Williams et al., 20151998; Forrier y Sels, 2003; Llinares, Zacarés y Córdoba, 2016). 
En esta línea, la empleabilidad es vista como un concepto complejo que incorpora algunas dimensiones personales, pero también algunas dimensiones contextuales y el ajuste dinámico entre unas y otras (McQuaid y Lindsay, 2005; Suárez, 2016; Llinares Insa, Zacarés González y Córdoba Iñesta, 2016). Por ejemplo, la propia visión de las competencias como un atributo personal puede ser revisada para incorporar las competencias, también, como un rasgo o característica de los grupos, equipos y comunidades (Boreham, 2004), lo que nos lleva a poner el acento en los procesos sociales que ocurren en los contextos laborales. En esta visión amplia del concepto de empleabilidad es necesario incluir los factores contextuales y las llamadas “condiciones de efectividad” (De Grip, Loo, y Sanders, 2004) que se definen como factores específicos del contexto que ayudan, o dificultan, la empleabilidad de las personas (por ejemplo, la oferta formativa de la empresa).

Este intento de integrar diferentes elementos se aprecia, por ejemplo, en la definición que proponen De Grip et al. (2004: 216): "La empleabilidad se refiere a la capacidad y disposición de los trabajadores para seguir siendo atractivos para el mercado laboral (factores de oferta), reaccionando y anticipando cambios en las tareas y el entorno laboral (factores de demanda), facilitados por los instrumentos de desarrollo de recursos humanos disponibles (instituciones)". En esta línea, Thijssen, Van der Heijden y Rocco (2008) proponen una “definición estratificada” en tres niveles. Una definición básica relativa a la capacidad individual para desempeñar diferentes puestos en un mercado de trabajo; una definición más amplia que añade la capacidad de mejorar y gestionar la propia trayectoria profesional; y una definición completa, que incluye todos los factores individuales y contextuales que influirán en la posición en un mercado de trabajo determinado.

En síntesis, para mejorar la empleabilidad de personas en situación o riesgo de exclusión es imprescindible adoptar una perspectiva amplia (recogiendo el mayor número de elementos), que sea dinámica (para incorporar los cambios que se producen en el tiempo) e interactiva (enfatizando la relación entre los diferentes elementos). En este sentido podemos definir la empleabilidad como el conjunto de factores y condiciones tanto individuales como contextuales $-y$ la relación entre ellos- que influyen en que una persona puede conseguir un empleo, mantenerlo y mejorarlo. En esta definición se busca mantener el equilibrio entre la responsabilidad personal y social y el alcance individual y colectivo.

Esta comprensión de empleabilidad tiene algunas implicaciones de cara al acompañamiento en las empresas de inserción:

- Comprender a las empresas de inserción desde su capacidad de crear entornos que promueven la empleabilidad a través de su propio funcionamiento, organización y nivel de competencia colectiva.

- La relevancia de desarrollar las conexiones y las relaciones de coordinación, cooperación y colaboración con el mercado de trabajo, de cara a facilitar la inserción de las personas trabajadoras de inserción y la mejora de la accesibilidad de esos entornos.

\section{Empresas de inserción y empleabilidad: definiendo el modelo}

\subsection{Empresas de inserción: respuesta a necesidades complejas de inserción laboral}

Las empresas de inserción, en el contexto del estado español son una iniciativa con un fuerte desarrollo en esta última década), habiéndose configurado en este período como una parte relevante de la economía social con el objetivo de dar respuesta a personas en situación o riesgo de exclusión social (Askunze, 2016; Díaz, Marcuello y Marcuello, 2012, Alvarez de Eulate, 2017).

El origen de las empresas de inserción a nivel europeo podemos situarlo en Francia en la década de los setenta, donde tienen un especial impacto y desarrollo (Urteaga, 2009) y se generalizan, más tarde a otros países de Europa y Norteamérica (Cooney, 2015; O’Connor y Meinhard, 2014). Las empresas de inserción se enmarcan en el conjunto de iniciativas de "inserción por lo económico" que incluyen una amplia gama de modalidades (Aguilar, Laparra y Corera, 1999; Coque y Pérez, 2000; Davister, Defourny y Gregoire, 2004; González Barriga, 2003): empresas de inserción transitorias, empleo social protegido, empresas sociales solidarias, empresas de trabajo temporal de inserción, empresas tuteladas, centros especiales de empleo o, incluso, centros ocupacionales. Este conjunto de iniciativas pretende abarcar diferentes situaciones de empleabilidad a través de poner el énfasis en alguna de las dimensiones del empleo (Méda, 1998) y, en consecuencia, se orientan a objetivos diversos. Estas iniciativas cubren un abanico que va desde programas orientados a la calidad de vida a través de la actividad laboral -centros ocupacionales- en los que la mejora de la empleabilidad juega un papel secundario hasta -empresas de trabajo temporal de inserción- para aquellas personas más cercanas al mercado de trabajo.

Las personas con dificultades en el mercado laboral se enfrentan con frecuencia a múltiples barreras al empleo que se solapan. Los enfoques que solo abordan algunos de estos obstáculos pueden no ser suficientes para facilitar el retorno al empleo (Fernandez et al., 2016). Se trata de personas que por diversos motivos han estado fuera del mercado laboral durante mucho tiempo, que disponen de una escasa cualificación laboral y que se encuentran 
sin posibilidades de que las empresas les ofrezcan oportunidades laborales. Además, las dificultades de acceso al empleo suelen estar relacionadas también con otras dimensiones de sus vidas como la salud, ingresos, vivienda, atención a menores o la ausencia de soportes sociales (Nga y Chan, 2015) que exigen un abordaje integral para superar las diferentes barreras (Blumenberg, 2002) y que incluya, por tanto, una variedad de apoyos (Nga y Chan, 2015). Hay algunas evidencias de que la combinación de programas es más efectiva (Ramos, Suriñach y Artís, 2009).

Los programas de transición al empleo -en los que podemos incluir las empresas de inserciónintegran diferentes componentes (Dunn et al., 2016): experiencia de trabajo en entorno real, ingresos económicos, formación, servicios de apoyo, supervisión del puesto de trabajo y búsqueda y mantenimiento del empleo en el mercado ordinario. Se espera que estos programas consigan mejorar la cualificación profesional y la experiencia laboral, y apoyar en la gestión de las dificultades de la vida para que las personas puedan conseguir un empleo permanente y de calidad en las empresas ordinarias.

En el contexto español la normativa que regula las empresas de inserción recoge estos planteamientos. La Ley 44/2007 de Empresas de Inserción (España, 2007) identifica la mejora de la empleabilidad como el objetivo de las empresas de inserción con la finalidad de acceder a un empleo ordinario señalando que "deberán tener servicios de intervención o acompañamiento para la inserción sociolaboral que faciliten su posterior incorporación al mercado de trabajo ordinario" (art. 4).
3.2. Modelo de empleabilidad en las empresas de inserción

El modelo que a continuación presentamos entiende la trayectoria de una persona en la empresa de inserción como un proceso de mejora de la empleabilidad en el que influyen factores contextuales e individuales (Gráfico 1). La idea central es que la interacción entre la persona que se incorpora a la empresa de inserción -con unos factores individuales de empleabilidad-y la oferta de la empresa de inserción -con unos factores organizacionales de empleabilidad-va generando una diversidad de oportunidades y experiencias a lo largo de las diferentes fases del acompañamiento que debe tener un impacto en términos de mejora de la empleabilidad.

En primer lugar, las oportunidades de inserción laboral están condicionadas por el contexto socioeconómico del territorio y por las diversas circunstancias del mercado de trabajo, que pueden reducir o amplificar las oportunidades desarrolladas en la empresa de inserción. Además, la empresa de inserción se convierte en un elemento contextual que, de manera congruente con el medio social, articula oportunidades formativas y sociales a través del empleo, de forma que puedan mejorar los factores individuales de empleabilidad. Podemos suponer que, dependiendo de las características, el funcionamiento y organización de la empresas de inserción (Retolaza, Roqueñi et al., 2007) el impacto en la empleabilidad será diferente. En este sentido, será muy importante identificar lo que antes hemos definido como "condiciones de efectividad" (De Grip et al., 2004). Por ejemplo, se ha observado que las empresas de inserción individualizadas,

Figura 1. Modelo de mejora de la empleabilidad en las empresas de inserción (EI)
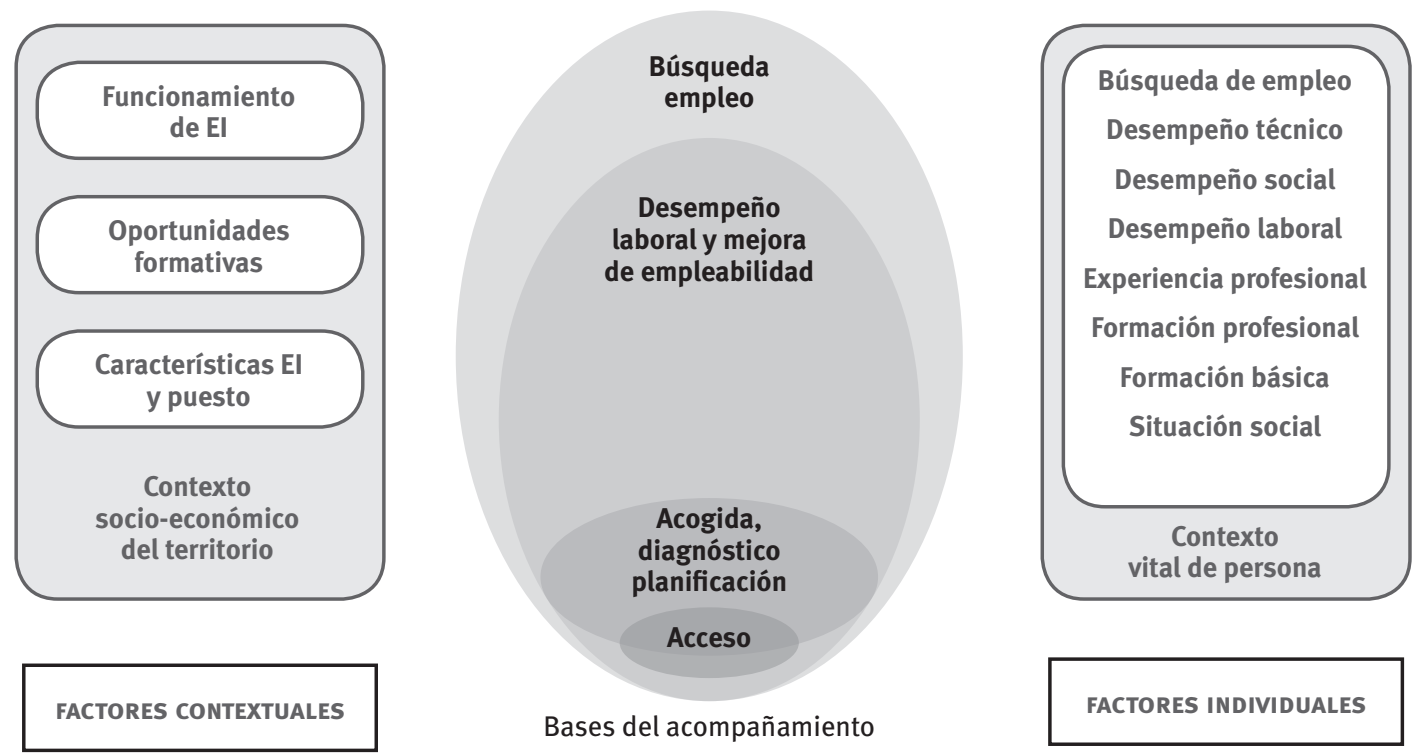

Fuente: Elaboración propia. 
flexibles y con un clima organizacional que da apoyo a las personas (O'Connor y Meinhard, 2014) logran mejores niveles de inserción en el empleo.

Por otra parte, las relaciones que mantiene la empresa de inserción con las empresas que se mueven en un determinado sector productivo van a ser una variable clave en las oportunidades laborales de las personas con contrato de inserción. Las conexiones que se den entre los puestos de trabajo de la empresa de inserción y los existentes en el mercado ordinario funcionan como una "cadena de movilidad" (Piore, 1983) que permite que un puesto de trabajo sirva como puerta de entrada a un conjunto de ocupaciones. En este sentido, la empleabilidad guardará relación con la existencia de puestos de trabajo que sirvan de puerta de entrada a un abanico amplio de puestos de trabajo (Miedes, 2002). 3.2.1. Factores organizacionales de empleabilidad: la
Escala de Factores Externos de Empleabilidad (CUFEE)

En este marco que venimos describiendo, resulta muy relevante identificar cuáles son los factores organizacionales de las empresas de inserción que influyen en la mejora de la empleabilidad. Aróstegui et al., (2018) han realizado un estudio² dirigido a identificar diferentes factores de las empresas de inserción que influyen en la empleabilidad y que se pueden evaluar a través de una escala: la Escala de Factores Externos de Empleabilidad (CUFEE) (Martínez-Rueda et al., 2018).

Tal como se presenta en la Tabla 1, la Escala CUFEE se compone de 3 dimensiones y 12 subdimensiones. La primera dimensión se refiere a las características generales de la empresa y a las relaciones y

Tabla 1. Dimensiones, subdimensiones e indicadores de CUFEE

\begin{tabular}{|c|c|c|}
\hline Dimensiones & Subdimensiones & Indicadores \\
\hline \multirow{3}{*}{$\begin{array}{l}\text { 1. Características } \\
\text { de la empresa } \\
\text { y del puesto de } \\
\text { trabajo }\end{array}$} & 1. Características de la empresa & $\begin{array}{l}\text { Desarrollo tecnológico, maquinaria y los útiles de trabajo, desarrollo } \\
\text { organizativo, exigencia productiva, manuales de procesos y procedimientos, } \\
\text { certificaciones de calidad. }\end{array}$ \\
\hline & $\begin{array}{l}\text { 2. Relaciones de la empresa de } \\
\text { inserción con el mercado de } \\
\text { trabajo }\end{array}$ & $\begin{array}{l}\text { Seguimiento salida de empresa de inserción, actividades de intermediación, } \\
\text { actividades de prospección, participan ferias o eventos sector, contacto de } \\
\text { empresas sector, empresa de inserción es conocida. }\end{array}$ \\
\hline & $\begin{array}{l}\text { 3. Conexiones del puesto de trabajo } \\
\text { con el mercado de trabajo }\end{array}$ & $\begin{array}{l}\text { Equivalentes en mercado ordinario y en catálogo cualificaciones, demanda } \\
\text { de ese tipo de puestos empleo encajado, empleo en empresa de inserción, } \\
\text { autoempleo. }\end{array}$ \\
\hline \multirow{3}{*}{$\begin{array}{l}\text { 2. Caracatersitcas } \\
\text { formativas del } \\
\text { puesto de trabajo }\end{array}$} & $\begin{array}{l}\text { 4. Oportunidades formativas del } \\
\text { puesto de trabajo }\end{array}$ & $\begin{array}{l}\text { Disposición de diferentes puestos, diferentes puestos en itinerario, diferentes } \\
\text { emplazamientos, desarrollo progresivo de competencias, formación previa a la } \\
\text { incorporación al puesto. }\end{array}$ \\
\hline & 5. Nivel de exigencia del puesto & $\begin{array}{l}\text { Ritmo, calidad (precisión, cuidado), responsabilidad, autonomía, iniciativa, } \\
\text { atención al público, trabajo en equipo. }\end{array}$ \\
\hline & $\begin{array}{l}\text { 6. Nivel de competencias exigibles } \\
\text { en el puesto }\end{array}$ & $\begin{array}{l}\text { Competencias personales, competencias sociolaborales, competencias } \\
\text { tecnicoprofesionales }\end{array}$ \\
\hline \multirow{6}{*}{$\begin{array}{l}\text { 3. Funcionamiento } \\
\text { de la empresa de } \\
\text { inserción }\end{array}$} & $\begin{array}{l}\text { 7. Sistematización de procesos de } \\
\text { trabajo }\end{array}$ & $\begin{array}{l}\text { Formalización de procedimientos de trabajo, sistematización de los procesos } \\
\text { productivos, recursos de apoyo al desempeño, instrumentos revisión de } \\
\text { tareas. }\end{array}$ \\
\hline & $\begin{array}{l}\text { 8. Ambiente, trabajo en equipo y } \\
\text { apoyo compañeros }\end{array}$ & $\begin{array}{l}\text { ambiente de trabajo colaborativo, apoyo entre compañeros, relaciones } \\
\text { personales, PTI con experiencia tienen funciones de apoyo, desarrollo de } \\
\text { apoyo entre compañeros, oportunidades de desafío, oportunidades de } \\
\text { observar otros puestos. }\end{array}$ \\
\hline & 9. Coordinación profesionales & $\begin{array}{l}\text { Visión compartida, definición de funciones entre coordinación gerencia y } \\
\text { técnicos/as de producción y acompañamiento. }\end{array}$ \\
\hline & $\begin{array}{l}\text { 10. Conciencia, regulación y } \\
\text { participación }\end{array}$ & $\begin{array}{l}\text { Reuniones regulares con PTI, espacio de participación PTI, procedimientos } \\
\text { situaciones de crisis, frecuencia de uso plan personal, PTI explican sentido, } \\
\text { conciencia de objetivos y fases. }\end{array}$ \\
\hline & 11. Acciones formativas & $\begin{array}{l}\text { Orientación formativa de PTP, desarrollan acciones formativas formales, } \\
\text { enseñanza específica tareas del puesto, participación en acciones externas, } \\
\text { acreditación de competencias. }\end{array}$ \\
\hline & $\begin{array}{l}\text { 12. Supervisión y apoyos al } \\
\text { desempeño laboral }\end{array}$ & $\begin{array}{l}\text { Supervisión directa y regular de tareas, consulta dudas y dificultades, uso } \\
\text { adaptaciones o ayudas técnicas, retroalimentación del desempeño laboral, } \\
\text { reconocimiento de desempeño, apoyo problemáticas ajenas al puesto, } \\
\text { necesidades no laborales, informados de planes personales, protagonismo en } \\
\text { planes personales. }\end{array}$ \\
\hline
\end{tabular}

Fuente: Elaboración propia.

${ }^{2}$ Estudio realizado en el marco del proyecto de investigación "Procesos de formación, acompañamiento, cualificación y desarrollo personal en empresas de inserción: innovación social desde el empleo" (EDU2013-45919-R), financiado por el Ministerio de Economía y Competitividad. 
vinculaciones que tanto la empresa como el puesto de trabajo mantienen con el mercado de trabajo ordinario. La segunda dimensión incluye aquellos aspectos relacionados con las características formativas que ofrecen los puestos de trabajo e incorpora elementos como las oportunidades formativas de los puestos, los niveles de exigencia y las competencias que ponen en juego.

La tercera dimensión -Funcionamiento de la empresa de inserción - incluye los aspectos "micro" que tienen que ver con el desarrollo de las actividades y las interacciones en el discurrir cotidiano de las empresas, y aspectos relacionados con el grado de sistematización de los procesos productivos, el clima, el grado de coordinación o las formas de supervisión y apoyos a las personas.

\subsubsection{Factores individuales de empleabilidad: la Escala de Factores de Empleabilidad (EFE)}

En el marco del trabajo de revisión del Manual de Acompañamiento en las Empresas de Inserción, se ha desarrollado otro estudio dirigido a identificar aquellos factores individuales que influyen en la empleabilidad y que son relevantes para la intervención en las empresas de inserción. Producto de ese trabajo es la Escala de Factores de Empleabilidad (EFE) que se encuentra actualmente en fase de validación. El objetivo de la EFE es evaluar la situación de una persona con relación a los diferentes factores que se asocian a la empleabilidad con la finalidad de definir o mejorar los servicios y apoyos que esa persona recibe en su itinerario de inserción laboral.

En esta escala se exploran los factores individuales -incluyendo las circunstancias en las que se encuentran las personas- teniendo en cuenta las oportunidades del contexto. En este sentido, aunque el conjunto de factores que se exploran hacen referencia a las personas, es importante señalar que siempre se trata de ubicarlos en el contexto en el que se producen. Todos se refieren a la situación de una persona, pero sin perder de vista que la situación de esa persona está influida de forma permanente por el contexto en el que se encuentra. Además, y en la medida en que quiere servir de referencia en la elaboración de planes de empleo, adopta un enfoque funcional y orientado a los resultados, porque evalúa factores que se centran en la situación y funcionamiento actual de la persona, que puede ser mejorado con el uso sistemático de apoyos y prestaciones.

La escala identifica ocho dimensiones que se definen en la Tabla 2.

\section{La oferta de las empresas de inserción: clarificando las prestaciones}

\subsection{La oferta de las empresas de inserción}

La oferta de las empresas de inserción, dirigida a personas con especiales dificultades para acceder al empleo, se articula en torno a proporcionar un empleo que sirva de base para el desarrollo de un proceso-itinerario de inclusión social y laboral.

En este sentido, ofrece un conjunto amplio de oportunidades a personas que, por diferentes razones, han sido excluidas de oportunidades formativas, laborales o sociales. Las personas con especiales dificultades de acceso al empleo suelen enfrentar obstáculos de muy diferente tipo para poder incorporarse a un mercado de trabajo cada vez más exigente y competitivo. Además de un conjunto de circunstancias personales con dificultades en aspectos como salud, ingresos, vivienda o apoyo

Tabla 2. Dimensiones de la Escala de Factores de Empleabilidad (EFE)

\begin{tabular}{|c|c|}
\hline Dimensión & Definición \\
\hline 1. Situación social & $\begin{array}{l}\text { Conjunto de circunstancias personales y sociales que influyen en que la persona pueda encontrar un } \\
\text { empleo. Incluye las posibilidades de conciliación, situación legal, ingresos, vivienda, redes de apoyo, } \\
\text { salud física, salud mental, acceso al transporte y riesgo de discriminación. }\end{array}$ \\
\hline 2. Formación básica & $\begin{array}{l}\text { Nivel de cualificación personal básica que incluye el conocimiento de idioma, competencias básicas } \\
\text { comunicativas y sociales, titulaciones académicas y el uso de tecnologías de la información y la } \\
\text { comunicación. }\end{array}$ \\
\hline 3. Formación profesional & $\begin{array}{l}\text { Niveles y características de la formación profesional, tanto reglada como para el empleo, incluyendo } \\
\text { la situación actual con relación a la formación. }\end{array}$ \\
\hline 4. Experiencia profesional & $\begin{array}{l}\text { Duración y características de la trayectoria laboral de la persona (coherencia, tiempo en desempleo, } \\
\text { etc.). }\end{array}$ \\
\hline 5. Búsqueda de empleo & $\begin{array}{l}\text { Factores relacionados con el afrontamiento de la búsqueda de empleo; incorpora aspectos como el } \\
\text { grado de prioridad del empleo, proyecto profesional, información del mercado de trabajo, redes de } \\
\text { apoyo, disponibilidad, gestión de plan de búsqueda y empleabilidad percibida. }\end{array}$ \\
\hline 6. Desempeño sociolaboral & $\begin{array}{l}\text { Competencias sociales básicas necesarias para cualquier empleo, tales como comunicación, } \\
\text { relaciones, trabajo en equipo, autocontrol y defensa de derechos. }\end{array}$ \\
\hline $\begin{array}{l}\text { 7. Desempeño social en el puesto } \\
\text { de trabajo }\end{array}$ & $\begin{array}{l}\text { Competencias básicas relacionadas con las tareas y con las normas de funcionamiento en el empleo } \\
\text { (imagen personal, normas y hábitos de trabajo, responsabilidad, autonomía, iniciativa y autoeficacia). }\end{array}$ \\
\hline 8. Desempeño técnico profesional & $\begin{array}{l}\text { Las competencias tecnicoprofesionales vinculadas con cada puesto de trabajo. Son específicas de } \\
\text { cada puesto de trabajo u ocupación. }\end{array}$ \\
\hline
\end{tabular}


social, frecuentemente han sido excluidos de procesos formativos, de cualificación profesional o de oportunidades de empleo. El impacto de este conjunto de circunstancias hace que, en muchas ocasiones, la capacidad de las personas para gestionar su propia vida se vea limitada, afectando a su capacidad de autodeterminación, contribuyendo a cerrar un círculo de falta de oportunidadescircunstancias personales-limitaciones personalesindefensión aprendida.

Para romper este círculo hace falta una apuesta comprometida por modificar, en primer lugar, las condiciones del contexto, del ambiente de las personas. En este sentido, las empresas de inserción ofrecen una buena oportunidad al tomar como punto de partida la creación de mejores condiciones en la vida de las personas a través de ofrecer un empleo, con todo lo que implica, y un conjunto de prestaciones o apoyos asociados a él.

De cara a clarificar las oportunidades que ofrecen las empresas de inserción, es relevante identificar los diferentes ámbitos que configuran su oferta:

- Empleo remunerado. En primer lugar, participar en una empresa de inserción permite disponer de unos ingresos con cierta continuidad, lo que ya de por sí supone una oportunidad para las personas de cara a "ordenar" algunas dimensiones de su vida. En algunas situaciones, y para facilitar que la persona pueda participar en procesos productivos, puede ser necesario poner en marcha medidas de accesibilidad, adaptaciones de puestos 0 ajustes.

- Desarrollo psicosocial. Desarrollar una actividad regular con sentido y proyección social tiene una influencia importante en la vida de las personas: estabilidad personal, ocupación y estructuración del tiempo, relaciones sociales, sentimiento de utilidad y pertenencia. Al mismo tiempo, el hecho de que las personas en situación o riesgo de exclusión hayan experimentado durante un tiempo prolongado situaciones de fracaso, pobreza, inactividad o aislamiento social tiene un impacto en su capacidad de afrontar retos en su vida y de desarrollar sus proyectos vitales.

- Formación. Es un elemento diferenciador de las empresas de inserción en la medida en que las personas que trabajan en ellas están en un proceso de mejora de su cualificación. Esta cualificación tiene, a su vez, algunas características particulares:

- Combina formación en el puesto, uno de sus aspectos centrales, con otros procesos formativos (formación académica, acreditación profesional, formación profesional, formación para la vida y la ciudadanía, etc.).

- Incluye competencias tanto tecnicoprofesionales como sociolaborales y personales.

- En la medida en que las personas están en situación de exclusión, es relevante incorporar aspectos de educación ciudadana, transformadora y para la emancipación.

- Acompañamiento social. Además de los aspectos anteriores, relacionados más o menos directamente con la realización de una actividad laboral, las empresas de inserción tienen el encargo de apoyar a las personas en la mejora de otras dimensiones (salud, derechos, vivienda, etc.) no laborales, pero que tienen gran impacto en el bienestar de las personas, y también en su empleabilidad, en la medida en que constituyen una condición de posibilidad para el desarrollo de procesos de inclusión laboral.

\section{- Promoción personal y transformación} social. Las empresas de inserción han de ser "organizaciones de participación" en la medida en que la "participación es un instrumento de inserción”, por lo que el nivel que alcanzan las personas es un indicador de su nivel de inclusión (Coque y Pérez, 2000). La participación es tanto su producto esencial (las personas con dificultades consiguen insertarse en la sociedad conforme participan más y de modo más complejo) como el eje del proceso para conseguir dicho producto (se aprende a participar participando, paulatinamente, cada vez más) (Coque y Pérez, 2000). Además de los aspectos anteriores, dirigidos más a las personas, las empresas de inserción también desarrollan un trabajo desde el punto de vista de incidir en las condiciones que generan exclusión laboral tanto en su propio funcionamiento (organización y formas de participación, accesibilidad, etc.) como en el mercado laboral.

\subsection{Prestaciones y apoyos: ¿qué dice la normativa?}

Tal como hemos señalado en un apartado anterior, la normativa tanto estatal como vasca identifica la mejora de la empleabilidad como el objetivo de las empresas de inserción con la finalidad de acceder a un empleo ordinario. Aunque con diferentes matices, ambas normativas definen las medidas de intervención y acompañamiento, en el marco de itinerarios de inserción sociolaboral, entendidos como:
"El conjunto de servicios, prestaciones, acciones de orientación, tutoría y procesos personalizados y asistidos de trabajo remunerado, formación en el puesto de trabajo, habituación laboral y social encaminados a satisfacer o resolver problemáticas específicas derivadas de la situación de exclusión que dificultan a la persona un normal desarrollo de su itinerario en la empresa de inserción" (Ley 44/2007, art. 3; Decreto 182/2008, art. 6).

El Decreto 182/2008 también concreta (art.10) esas medidas personalizadas de apoyo:

\footnotetext{
“-Una formación dirigida al aprendizaje de una determinada actividad profesional y a la adecuación del nivel formativo o las competencias
} 
profesionales a las exigencias del mercado laboral.

-El establecimiento de unas pautas de funcionamiento destinadas a la adquisición de hábitos sociales y de trabajo.

-Un servicio de acompañamiento social, encaminado a satisfacer o resolver problemáticas personales y de convivencia que impiden o dificultan el normal funcionamiento en el empleo. -Un servicio de acompañamiento en la fase final de tránsito" (Gobierno Vasco, 2008).

Del análisis de la normativa podemos identificar las prestaciones o apoyos que la empresa de inserción debe desarrollar: trabajo asistido; habituación laboral y social; formación en el puesto de trabajo; formación para la adecuación del nivel formativo/ competencias a las exigencias del mercado; servicios de acompañamiento social; servicios de acompañamiento en la fase de transición.

\subsection{Finalidades y áreas de apoyo}

La finalidad de la empresa de inserción es mejorar la empleabilidad de una persona, entendida de forma global e interactiva - factores internos y externospara que esté en mejores condiciones de conseguir y mantener un empleo ordinario que mejore su calidad de vida.

En este sentido, los resultados esperables de la participación de una persona en una empresa de inserción podrán ser:
- Incrementar de forma significativa las condiciones sociales y circunstancias personales que influyen en su empleabilidad.

- Disponer de una cualificación sociolaboral y de cualificación tecnicoprofesional con reconocimiento en el mercado laboral.

- Disponer de un plan de empleo orientado a la inserción en el mercado ordinario.

- Percibirse con capacidad de transformar su entorno y con estrategias para gestionar su propia vida y defender sus derechos.

Para conseguir estos resultados, la empresa de inserción se relaciona tanto con la persona como con el mercado de trabajo con el objetivo de incidir en los factores de empleabilidad. En este sentido, también se espera que la empresa desarrolle procesos de prospección, intermediación, apoyo en la búsqueda, colocación y mantenimiento de un empleo en colaboración con los mercados de trabajo locales.

La empresa de inserción desarrolla un proceso básico, transversal al resto de procesos, de diagnósticoplanificación que guía el proceso de la persona y cinco procesos clave: apoyos a la actividad laboral, cualificación profesional, acompañamiento social, promoción personal-empoderamiento e inserción en empleo ordinario (Figura 2). Estos seis procesos definen lo que denominamos "áreas o ámbitos de apoyo", en cada una de las cuales vamos a identificar la prestación que se realiza y las acciones o apoyos específicos que la componen (Tabla 3).

Figura 2. Prestaciones para la empleabilidad en la empresa de inserción

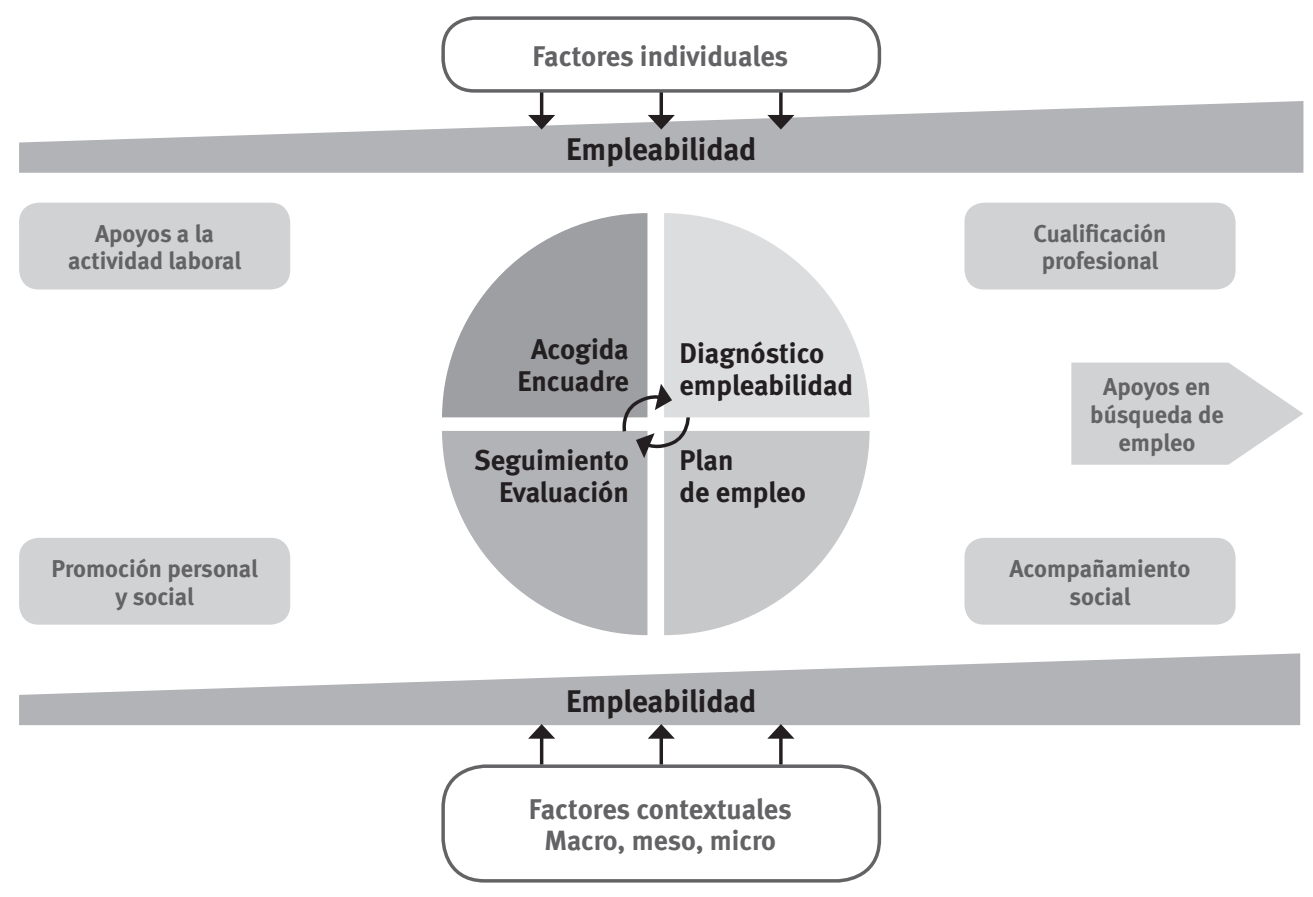

Fuente: Elaboración propia. 
Proceso Básico: diagnóstico de empleabilidad y plan de empleo

Este primer ámbito de apoyo es el proceso básico de apoyo a las personas y toma como referencia la evaluación de la situación de la persona con relación a la empleabilidad y la gestión del propio itinerario, identificando sus recursos personales y los apoyos disponibles para conseguir un empleo de calidad y mantenerlo.

Las prestaciones que se corresponden en este ámbito son:

- Diagnóstico de empleabilidad, que incorpora las necesidades identificadas.

- Plan personal de empleo, que en diálogo con la persona incorpora sus metas y concreta los recursos y apoyos que se van a utilizar.

- Acompañamiento del plan de empleo que supone el desarrollo, a través de la participación de la persona en la empresa de inserción, de una relación de ayuda para la conciencia de situación, toma de decisiones, resolver problemas, acceder a recursos y construir vínculos.

\section{Proceso clave 1. Apoyo a la actividad laboral}

Para aquellas personas que han estado alejadas durante mucho tiempo de la formación o del mercado laboral, desempeñar un puesto de trabajo o realizar una actividad laboral con continuidad y exigencia puede requerir de determinados apoyos. Aunque puede incluir medidas o apoyos diversos, todos ellos se dirigen a crear las condiciones que permiten que la persona pueda desarrollar una actividad productiva de forma regular. Los apoyos pueden incluir flexibilidad horaria, adaptación de puestos o apoyo de otros trabajadores para asegurar que la persona desarrolle la actividad laboral, y entendiendo que ello configura una prestación diferenciada: el apoyo a la actividad laboral.

\section{Proceso clave 2. Cualificación profesional}

La formación profesional, junto con el acompañamiento social, conforman los aspectos centrales de las prestaciones de las empresas de inserción. La formación se dirige a mejorar las competencias profesionales y a que se adecuen a las exigencias del mercado laboral. En la medida en que en las empresas de inserción se desarrolla un trabajo productivo, la principal prestación formativa se relaciona con la formación en el propio puesto de trabajo. En este sentido, las propias características de los puestos de trabajo disponibles, su relación con el mercado de trabajo y el nivel de competencias implicadas son aspectos clave que van a marcar la calidad de la oferta formativa. En cualquier caso, las estrategias 0 acciones propias de la formación en el puesto, tales como observación, evaluaciones de desempeño o enseñanza incidental, son algunos de sus componentes. La formación en el puesto, aun siendo clave en las empresas de inserción, tiene o puede ser complementada con otra formación, bien en la propia empresa o participando en procesos formativos externos.

Dada la diversidad de las situaciones formativas que podemos encontrarnos en las empresas de inserción, puede ser interesante considerar los diferentes perfiles de entrada-salida en la vertiente formativa. En este sentido, la triple dimensión de las competencias (técnica, sociolaboral y personal) puede tener diferentes niveles de logro o expectativa. La dimensión técnica se regula por los "niveles de cualificación" en las diferentes familias profesionales. La dimensión sociolaboral también puede tener diferentes niveles, con un nivel básico referido a la "habituación laboral" y otras competencias más complejas o de nivel avanzado referidas a la forma de afrontar las tareas y las relaciones en el entorno laboral. Por último, las competencias personales incluyen otros aspectos relacionados con las circunstancias personales y no tanto con el entorno laboral.

En síntesis, identificamos tres prestaciones en el ámbito de la formación que es deseable que se integren en una propuesta de itinerario formativo:

- Formación en el puesto. Incluye el aprendizaje informal-incidental (Marsick y Watkins, 2001) que se produce en el marco del desempeño laboral y de los procesos productivos. Es deseable que tenga referencias en el catálogo de cualificaciones profesionales y en los sistemas de acreditación profesional (Guillera y Chisvert, 2018). Además, hay que tener en cuenta que se trata de un aprendizaje de tipo informal-incidental (Marsick y Watkins, 2001). Aunque no se trata de un aprendizaje formal, es interesante explicitar las condiciones mínimas que se deben dar para que produzca una mejora de la empleabilidad. Esto tiene que ver con sistematizar lo informal, es decir, con la creación de espacios, tiempos, agrupamientos y roles que se pueden crear para que se optimicen los procesos de aprendizaje que, de hecho, se producen producto de la participación de las personas en un determinado proceso productivo. Al mismo tiempo, son muy importantes los aspectos implícitos, tales como creencias, suposiciones, interpretaciones 0 normas sociales, que se van construyendo en el funcionamiento cotidiano de la empresa.

- Formación interna. Incluye las acciones formativas complementarias, como por ejemplo cursos, seminarios, talleres o material de autoaprendizaje, que se organizan desde la propia empresa para responder a las necesidades de formación de sus trabajadores y trabajadoras.

- Formación externa. Se refiere a las actividades de orientación, mediación y apoyo para que las personas con contrato de inserción puedan 
participar en programas formativos impartidos por entidades ajenas a las empresas de inserción.

\section{Proceso clave 3. Acompañamiento social}

Como hemos señalado, la mejora de la empleabilidad se relaciona, en personas con especiales dificultades de acceso al mercado laboral, con diversas circunstancias personales que son las que permiten o dificultan la participación de una persona en el empleo. En ese sentido, la mejora de dicha empleabilidad tiene que basarse en la creación de unas condiciones vitales mínimas que permitan a la persona poner en el centro su proceso de inserción laboral y sentirse protagonista de él.

Por acompañamiento social entendemos el proceso de ponerse a disposición de la persona, de cara a conseguir resultados significativos para ella en las diferentes dimensiones vitales tales como vivienda, situación legal/administrativa, apoyos sociales, movilidad, salud o ingresos, y que están incidiendo en su probabilidad de encontrar y mantener un empleo.

Por tanto, en este ámbito, la prestación consiste en el acompañamiento social que ofrece apoyos personales (Wehmeyer et al., 2010) de diferente intensidad, que contribuyen a mejorar la situación y circunstancias personales en las diferentes dimensiones vitales, como condición de posibilidad de itinerarios laborales exitosos. Dependiendo de la situación y de sus propias expectativas, este apoyo puede darse en alguna o varias de esas dimensiones vitales.

\section{Proceso clave 4. Promoción personal, social y cultural}

Aunque es transversal a los otros procesos, su relevancia y la especificidad de su desarrollo nos llevan a identificarla como un ámbito diferenciado de intervención. En cierta medida es el eje, el que da sentido a los demás y que permite integrarlos en un proyecto de una vida digna y en el que emerge la persona, como miembro de una comunidad, con capacidad de lenguaje (decir la palabra) y acción (transformar la realidad).
En este sentido, promover -creando condiciones-, el protagonismo de las personas en la gestión de sus propios asuntos vitales es una cuestión a tener presente de forma permanente. El "sentido de agencia" de las personas, su empoderamiento como agentes activos en su discurrir vital, apoyando la participación de la persona en sus diferentes entornos -en primer lugar, el propio laboral-, aumentando la frecuencia, tipo y calidad de las interacciones sociales que mantiene. Se dirige a promover la autodeterminación (conciencia de fortalezas y debilidades, elecciones y toma de decisiones, elaboración de planes, resolución de problemas, defensa de derechos, etc.) como el elemento central de todo el proceso de acompañamiento a las personas.

En este sentido, resulta relevante identificar una prestación como "apoyo al empoderamiento y la participación social” que puede tener acciones o apoyos diferentes, pero que tienen en común una manera particular de ordenar el medio y de desarrollar todas ellas. Enriquecer el medio a través de promover canales de participación, de construir grupos humanos inclusivos, espacios colectivos de reflexión y diálogo o apoyo psicosocial son componentes de esta prestación.

\section{Proceso clave 5. Búsqueda y apoyo en empleo ordinario}

Por último, y en la medida en que el acceso a un empleo ordinario es la finalidad de la empresa de inserción y requiere de unas actuaciones profesionales específicas, una prestación imprescindible tiene que ver con el desarrollo de un proceso de apoyo para conseguir un empleo. Las prestaciones implicadas son la elaboración de un plan de búsqueda activa de empleo y los apoyos en el mantenimiento del empleo que pueden incluir acciones o apoyos tales como orientaciónplanificación, intermediación, mediación, apoyo en la búsqueda, apoyo en colocación y apoyo al mantenimiento.

En la Tabla 3 se presenta un cuadro síntesis que resume la propuesta de prestaciones y apoyos atendiendo al ámbito de acción, la identificación de las prestaciones, así como su definición y contenido. 
Tabla 3. Clasificación de las prestaciones y apoyos de acompañamiento en las empresas de inserción

\begin{tabular}{|c|c|c|c|}
\hline Proceso & Prestaciones & Definición & Contenido \\
\hline $\begin{array}{l}\text { Diagnóstico y plan } \\
\text { de empleo }\end{array}$ & $\begin{array}{l}\text { - Diagnóstico de } \\
\text { empleabilidad. } \\
\text { - Plan de Empleo. } \\
\text { - Acompañamiento en el plan } \\
\text { de empleo. }\end{array}$ & $\begin{array}{l}\text { Valoración con la persona de } \\
\text { su situación de empleabilidad } \\
\text { y el apoyo en la elaboración y } \\
\text { seguimiento de su plan de empleo. }\end{array}$ & $\begin{array}{l}\text { Gestión del propio itinerario, identificando sus } \\
\text { recursos personales y los apoyos disponibles } \\
\text { para conseguir un empleo de calidad y } \\
\text { mantenerlo. }\end{array}$ \\
\hline Desempeño laboral & $\begin{array}{l}\text { - Apoyos a la actividad } \\
\text { laboral. }\end{array}$ & $\begin{array}{l}\text { Diseño de adaptaciones y apoyos } \\
\text { que facilitan el desarrollo de una } \\
\text { actividad laboral de forma regular. }\end{array}$ & $\begin{array}{l}\text { Condiciones que permiten que la persona puede } \\
\text { desarrollar una actividad productiva de forma } \\
\text { regular. }\end{array}$ \\
\hline $\begin{array}{l}\text { Cualificación } \\
\text { profesional }\end{array}$ & $\begin{array}{l}\text { - Formación en el puesto. } \\
\text { - Formación interna (cursos, } \\
\text { acciones formativas, online). } \\
\text { - Formación externa (cursos, } \\
\text { carnets, etc.). }\end{array}$ & $\begin{array}{l}\text { Desarrollo de acciones diversas } \\
\text { dirigidas a la mejora de las } \\
\text { competencias profesionales. }\end{array}$ & $\begin{array}{l}\text { La cualificación técnica toma como referencia } \\
\text { los niveles de cualificación asociados a } \\
\text { determinadas ocupaciones. } \\
\text { La cualificación sociolaboral incluye } \\
\text { componentes básicos asociados a la } \\
\text { habituación laboral (realizar tareas, normas de } \\
\text { funcionamiento, desenvolvimiento laboral, etc.), } \\
\text { pero también otras más avanzadas relacionadas } \\
\text { con la iniciativa, trabajo en equipo, etc. }\end{array}$ \\
\hline $\begin{array}{l}\text { Acompañamiento } \\
\text { social }\end{array}$ & - Acompañamiento social. & $\begin{array}{l}\text { Conjunto de acciones centradas en } \\
\text { la persona que promueven el logro } \\
\text { de metas personales en diferentes } \\
\text { dimensiones de calidad de vida y } \\
\text { de acceso a la ciudadanía. }\end{array}$ & $\begin{array}{l}\text { El acompañamiento implica un proceso de } \\
\text { clarificación, toma de decisiones, búsqueda de } \\
\text { apoyos y recursos. } \\
\text { En función de la situación de las personas, de } \\
\text { sus intereses y objetivos, puede referirse al } \\
\text { conjunto de dimensiones o a alguna de ellas } \\
\text { y puede implicar apoyos de diverso tipo e } \\
\text { intensidad. }\end{array}$ \\
\hline $\begin{array}{l}\text { Promoción } \\
\text { personal, social y } \\
\text { cultural }\end{array}$ & $\begin{array}{l}\text { - Apoyo al empoderamiento y } \\
\text { la participación social. }\end{array}$ & $\begin{array}{l}\text { Dinamización, participación, } \\
\text { incidencia, comunidad. }\end{array}$ & $\begin{array}{l}\text { Toma de decisiones, autodeterminación, sujeto } \\
\text { activo, locus de control, sentido de agencia, } \\
\text { integración relacional. }\end{array}$ \\
\hline $\begin{array}{l}\text { Búsqueda y } \\
\text { apoyo en empleo } \\
\text { ordinario }\end{array}$ & $\begin{array}{l}\text { - Plan de búsqueda activa de } \\
\text { empleo. } \\
\text { - Apoyo a la búsqueda y } \\
\text { mantenimiento del empleo. }\end{array}$ & $\begin{array}{l}\text { Conjunto de acciones, incluyendo } \\
\text { un plan de búsqueda de empleo, } \\
\text { para apoyar que una persona puede } \\
\text { acceder y mantener un empleo de } \\
\text { calidad. }\end{array}$ & $\begin{array}{l}\text { Acciones de apoyo a la transición a un empleo } \\
\text { ordinario. }\end{array}$ \\
\hline
\end{tabular}

\section{Conclusiones}

Las empresas de inserción están demostrando ser una herramienta de gran potencialidad para abordar las necesidades de empleo de aquellas personas con necesidades complejas de inserción laboral. Una de sus aportaciones es permitir un enfoque integral que supera los enfoques centrados en las personas para adoptar una perspectiva holística con incidencia tanto en diferentes dimensiones vitales de las personas como en algunos de los aspectos contextuales que influyen en la falta de oportunidades que experimentan.

En este artículo hemos presentado una propuesta que operativiza estos planteamientos tanto proponiendo algunas herramientas que están siendo ensayadas como definiendo las prestaciones y apoyos que se pueden desarrollar desde las empresas de inserción. Esperamos que este esfuerzo de sistematización contribuya a mejorar los procesos de acompañamiento en ellas. 


\section{Referencias bibliográficas}

AGUILAR, M.; LAPARRA, M. y CORERA, C. (1999): “La incorporación sociolaboral en el Plan de Lucha contra la Exclusión en Navarra", Documentación Social, 117-118, 189-236.

ÁLVAREZ DE EULATE, L. (2017): “Empresas de inserción vascas: una herramienta eficaz y eficiente para la inclusión sociolaboral", Zerbitzuan, 64, 189201, 〈https://doi.org/10.5569/1134-7147.64.13〉.

ARÓSTEGUI, I.; ABIÉTAR, M.; MARTÍNEZ-RUEDA, N., y GALARRETA, J. (2018): “Analysis of external factors of employability in Work Integration Social Enterprises", en I. Academy (Ed.), 12th International Technology, Education and Development Conference, pp. 4.6144.620), Valencia, <https://doi.org/10.21125/ inted.2018>.

ASKUNZE, C. (2016): “Empresas de inserción en la economía social. Herramientas para la inclusión sociolaboral", Revista Jurídica, 29, 1-32, «www. ciriec-revistajuridica.es».

BECK, U. (1998): La Sociedad del Riesgo. Hacia una nueva modernidad, Barcelona, Paidos.

BLANCO, J.; KORTAJARENA, N. y ROMERO, V. (2015): Modelo Inclue, la apuesta por una atención de calidad en el ámbito de la inclusion social, Emaús, 〈www.emaus.com/informate/publicaciones〉.

BLUMENBERG, E. (2002): "On the Way to Work: Welfare Participants and Barriers to Employment", Economic Developmental Quartely, 16(4), 314-325, 〈https://doi. org/10.1177/089124202237196>.

BOREHAM, N. (2004): "A theory of collective competence: challenging the neo-liberal individualisation of performance at work", British Journal of Educational Studies, 52(1), 5-17, <http://firgoa. usc.es/drupal/files/Boreham - A Theory of Collective Competence.pdf〉.
COONEY, K. (2015): Social Enterprise in the United States: WISEs and Other Worker-Focused Models, Liege, «http://www.iap-socent.be/icsemproject>.

COQUE, J. y PÉREZ, E. (2000): Manual de creación y gestión de empresas de inserción social. Universidad de Oviedo, Oviedo, 〈http://www. caidosdelcielo.org/ong/wp-content/uploads/ MANUAL-EMPRESAS-INSERCION-SOCIAL.pdf〉.

DE GRIP, A.; LOO, J. VAN y SANDERS, J. (2004): “The Industry Employability Index: Taking account of supply and demand characteristics", International Labour Review, 143 (3), 211-233.

DÍAZ FONCEA, M.; MARCUELLO, C. y MARCUELLO, C. (2012): "Empresas sociales y evaluación del impacto social”, CIRIEC-España, Revista de Economía Pública, Social y Cooperativa, 75, 179-198, 〈http://www.ciriec-revistaeconomia.es/banco/ CIRIEC_7508_Diaz_et_al.pdf〉.

DONOSO, T. y FIGUERA, M.P. (2007): “Niveles de diagnóstico en los procesos de inserción y orientación profesional”, Revista Eletrónica de Investigación Psicoeducativa, 5 (11), 103-124, 〈http://www.investigacionpsicopedagogica. org/revista/articulos/11/espannol/Art_11_153. pdf $\rangle$.

DUNN, A.; MAXWELL, N.; ROTZ, D.; SHOJI, M.; GROVERROYBAL, C.; HU, M. y MCELROY, J. (2016): "Social Enterprises: Doing Good While Doing Business", Working Paper 42, Oakland.

FERNÁNDEZ, A.; GALARRETA, J. y MARTíNEZ, N. (2007): Manual de Acompañamiento en las Empresas de Inserción: Proceso y herramientas, Bilbao, Gizatea.

FERNANDEZ, R.; IMMERVOLL, H.; PACIFICO, D. y THÉVENOT, C. (2016): "Faces of Joblessness: Characterising 
Employment Barriers to Inform Policy", OECD Social, Employment and Migration Working Papers, 192, París, OECD, 〈https://doi. org/10.1787/5jlwvz47xptj-en〉.

FOESSA (2014): Informe sobre exclusión y desarrollo social en el País Vasco Resultados de la Encuesta sobre Integración y Necesidades, 〈http://www. caritasbi.org/cas/varios/informes/Informe_ FOESSA_Pais_Vasco_29-10-2014.pdf〉.

FORRIER, A. y SELS, L. (2003): "The concept of employability: a complex mosaic" International Journal Human Resources Development and Management, 3 (2), 102-124. <https://www.inderscienceonline. com/doi/abs/10.1504/IJHRDM.2003.002414〉.

GALLASTEGI, A., y MARTÍNEZ, N. (2011): La Mejora de los Procesos de Acompañamiento en las Empresas de Inserción, Bilbao, Gizatea.

GIZATEA (2018): Las Empresas de Inserción en el País Vasco. Memoria Social 2017, Bilbao, Gizatea.

ESPAÑA (2007): Ley 44/2007 para la regulación del régimen de las empresas de inserción, BOE, 299, 14 diciembre 2007, 〈https:// www.sepe.es/LegislativaWeb/verFichero. do?fichero=09017edb80039ed7〉.

PAÍS VASCO (2008): Decreto 182/2008 por el que se regula la calificación de empresas de inserción, se establece el procedimiento de acceso a las mismas y su registro, 〈https://www.euskadi. eus/bopv2/datos/2008/11/0806515a.pdf>.

GONZÁLEZ BARRIGA, J.R. (2003): “Introducción y Marco Jurídico de las Empresas de Inserción Laboral”, Acciones e Investigaciones Sociales, 18, 89-101.

GREGG, P. (2008): Realising Potential: A Vision for Personalised Conditionality and Support An independent report to the Department for Work and Pensions, 〈www.dwp.gov.uk/welfare〉.

GUILLERA, S. y CHISVERT, M.J. (2018): Acreditación de competencias profesionales. Guía para Empresas de Inserción, Madrid, Aeress y Faedei.

LLINARES, L.I.; ZACARÉS, J.J. y CÓRDOBA, A.I. (2016): "Discussing employability: current perspectives and key elements from a bioecological model", Employee Relations, 38 (6), 961-974, <https:// doi.org/10.1108/ER-07-2015-0145>.

MARSICK, V.J., y WATKINS, K.E. (2001): “Informal and Incidental Learning”, New Directions for Adult and Continuing Education, 89, 25-34. <https:// onlinelibrary.wiley.com/doi/abs/10.1002/ ace.5>.

MARTÍNEZ RUEDA, N., GALLASTEGI, A., y YÁNIZ, C. (2012): Evaluación y medición de competencias profesionales básicas en las empresas de inserción, Bilbao, Gizatea.

MARTÍNEZ RUEDA, N. y ALVAREZ DE EULATE, L. (2015): "Mejorando los procesos de acompañamiento en las empresas de inserción: una experiencia de colaboración entre Gizatea y la Universidad de Deusto", Lan Harremanak, 29(2), 236-244, <https://doi.org/10.1017/ CBO9781107415324.004>.

MARTÍNEZ-RUEDA, N.; GALARRETA, J. y AROSTEGUI, I. (2018): "Factores organizacionales que inciden en la mejora de la empleabilidad de las Empresas de
Inserción”, CIRIEC-España, Revista de Economía Pública, Social y Cooperativa, 94, 123-154, 〈https://doi.org/10.7203/CIRIEC-E.94.12697〉.

MCQUAID, R.W., y LINDSAY, C. (2005): “The concept of employability", Urban Studies, 42(2), 197-219, <https://doi.org/10.1080/004209804200031 6100>.

MÉDA, D. (1998): El trabajo. Un valor en peligro de extinción, Barcelona, Gedisa.

MIEDES, B. (2002): Empresas de inserción, el caso andaluz, Observatorio Local de Empleo, Universidad de Huelva.

NGA, A. y CHAN, W. (2015): "Social support for improved work integration Perspectives from Canadian social purpose enterprises", Social Enterprise Journal, 11(1), 47-68, 〈https://doi.org/10.1108/ SEJ-07-2014-0033>.

O'CONNOR, P. y MEINHARD, A. (2014): Work Integration Social Enterprises (WISES): Their Potential Contribution to Labour Market (Re-)Integration of At Risk Populations. Working Paper Series, Toronto, 〈http://www.ryerson.ca/cvss/ working_papers〉.

PÉREZ ERANSUS, B. (2015): “La evolución del empleo y del trabajo en un mundo globalizado . Cambios culturales y alternativas", Documentación Social, 178, 15-36.

PIORE, M. J. (1983): “Notas para una teoría de la estratificación del mercado de trabajo", en L. TOHARIA (comp.): El mercado de trabajo: teorías y aplicaciones (p. 193-221), Madrid, Alianza Editorial.

RAMOS, R.; SURIÑACH, J. y ARTÍS, M. (2009): “La efectividad de las políticas activas de mercado de trabajo para luchar contra el paro. La experiencia de Cataluña”, IREA Working Papers, 2009/19, 〈http://www.ub.edu/irea/working_ papers/2009/200919.pdf>.

RETOLAZA, J.L.; RUIZ, M. y ARAUJO, A. (2007): “Factores estratégicos de éxito de las empresas de inserción", CIRIEC-España, Revista de Economía Pública, Social y Cooperativa, 59, 61-89.

SANTOS ORTEGA A.; POVEDA ROSA M. (2002): Trabajo, Empleo y Cambio Social, Valencia, Tirant lo Blanch.

SCHALOCK, R.L. y VERDUGO, M.A. (2003): Calidad de Vida. Manual para profesionales de la educación, salud y Servicios Sociales, Madrid, Alianza.

SERRANO PASCUAL, A. (2000): "El concepto de empleabilidad en la estrategia europea de lucha contra el desempleo: Una perspectiva crítica”, Revista del Ministerio de Trabajo e Inmigración, 21, 137-150.

SIIS CENTRO DE DOCUMENTACIÓN Y ESTUDIOS (2012): Activación y derecho a la inclusión en el marco de las políticas de empleo y de garantía de ingresos en la CAPV/Aktibazioa eta gizarteratzako eskubidea, EAEko enpleguko eta diru-sarrerak bermatzeko politiken esparruan, Vitoria-Gasteiz, Gobierno Vasco, 〈https://www. siis.net/es/investigacion/ver-estudio/372/〉.

SUÁREZ, B. (2016): “Empleabilidad: análisis del concepto”, Revista de Investigación en Educación, 14(1), 67-84, 〈http://webs.uvigo.es/reined/〉. 
THIJSSEN, J.G.; VAN DER HEIJDEN, B.I. y ROCCO, T.S. (2008): "Toward the employability-link model: current employment transition to future employment perspectives", Human Resource Development Review, 7(2), 165-183.

URTEAGA, E. (2009): “La política de inserción por la economía en Francia", Acciones $e$ Investigaciones Sociales, 27, 195-216, 〈https://dialnet.unirioja.es/servlet/ articulo? codigo $=3171117$. .
WEHMEYER, M.L.; BUNTINX, W.H.E. et al., (2010): “El constructo de discapacidad intelectual y su relación con el funcionamiento humano", Siglo Cero, 39 (3), 5-18, «http://sid.usal.es/idocs/F8/ ART10928/constructo.pdf .

WILLIAMS, S.; DODD, L.; STEELE, C. y RANDALL, R. (2015): “A systematic review of current understandings of employability", Journal of Education and Work, 29(8), 877-901, <https://doi.org/10.1080/13639 080.2015.1102210〉. 